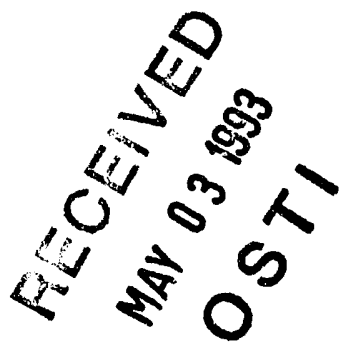

\title{
Wear-Mechanism Modelling
}

by

Michael F. Ashby

Cambridge University

Engineering Department

Trumpington Street

Cambridge CB2 1PZ, UK

Final Report

Argonne National Laboratory

Subcontract No. 90512401

March 1993
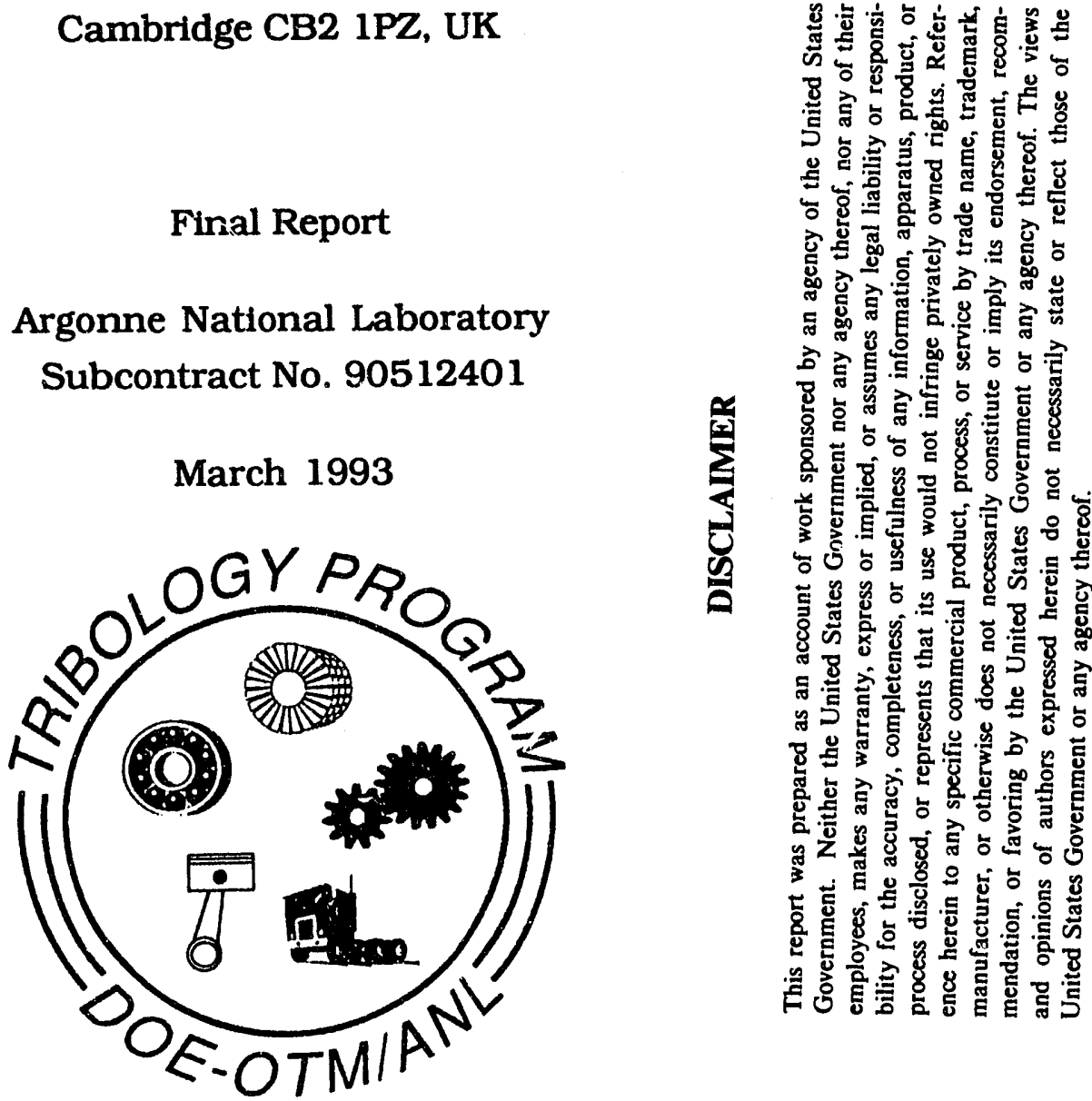

Prepared for:

U.S. Department of Energy

and

Argonne National Laboratory 


\author{
M.F. Ashby \\ Cambridge University Engineering Department, \\ Trumpington Street, \\ Cambridge CB2 1PZ, U.K.
}

August 1992

\title{
1. INTRODUCTION : Background and Goals
}

Wear, when two solids slide over each other, can involve a number of mechanisms. These include: wear by plastic processes, wear by brittle fracture caused by the contact force alone: brittle fracture induced by a combination of contact and thermal stresses; brittle fracture caused by adhesion in the stick-slip regime which appears when asperities on one surface reach their melting point, and wear caused by general melting. Evidence exists for all of these. It is obvious from this $l$ ist that the frictiona! heating induced by sliding is an important parameter: only the first two of che above mechanisms are possible if the surfaces remain coimpletely cold. The others all depend, to varying degrees, on a rise in the surface temperature. Surface temperature, then, is a helpful parameter: it combines some of the many variables of the problem, and it is one that can be calculated with useful accuracy. This, and an additional understanding of the way the contact pressure affects brittle fracture, allows a mechanism to be assigned to each regime of load and velocity (which, together with material properties, determine the surface temperatures $(1,2) *$ ), leading to the idea of a "wear-mechanism map". An example can be found in one of the publications arising from the work (3). The method has been applied with success to the interpretation of a number of applications in which friction either limits, or enhances, performance.

The main goals of the part of the DOE-OTM program at Cambridge were these:

* References are listed under "Deliverables" in Section 6. 
(a) to simplify and refine the calculation of surface temperatures in dry sliding, developing a sof tware tool (T-MAPS) which allows their efficient computation using widely-available hardware (IBM PC);

(b) to develop an experimental facility for pin-on-disk testing of ceramics which avoided the "overload" problems caused by inertia in dead-loaded machines; and to use this to generate friction and wear data;

(c) to carry out a broad survey of the present understanding of wear mechanisms in brittle solids, and to simplify, refine, or modify these as seemed appropriate;

(d) couple the temperature calculations with the models to give a firstat tempt at wear maps for brittle solids.

The success in achieving each goal is set out in Sections 2 to 5. Deliverables are listed in Section 6: Highlights in Section 7 . The research has drawn on a very large number of publications. No attempt is made to list these in this final report; they are fully referenced in the papers and reports arising from the research (Refs (1-5)), included with this final report.

\section{T-HAPS 4.0: A Softrare Tool for Surface Temperature Calculation}

Sliding with friction generates heat at the interface between sliding bodies. The heat is conducted into the bodies causing their temperature to rise. The temperature rise clepends on the mechanical and thermal properties of the bodies, on the load acting across the interface and on the velocity of sliding. In calculating these, it is essential to capture both the steady state (slow-sliding) and the transient (fast-sliding) limits, and the proper transition between them; and to distinguish between the "bulk" temperature and the "flash" temperature. The "bulk" temperature can be thought of as the average temperature of the surface beneath the nominal contact area of the slider: the "flash" temperature can be thought of as the short-lived peaks which appear at the contacting asperities which form the true surface contact area. At high loads, the two coincide; but at low loads the true contact area is much smaller than the nominal one, and the flash temperature is much higher than the bulk temperature. 
The T-MAPS 4.0 sof tware uses equations based on classical heat-flow to calculate these temperatures; details are given in references (1) and (2). The results are presented on the screen - and as hardcopy if desired - in the form illustrated by the first figure, which has been annotated to give details. The axes are the nominal contact-pressure, F/A $n$, in MPa ( $r$ ight) and sliding velocity, $v$, in $\mathrm{m} / \mathrm{s}$ (above); the other axes show contactpressure/hardness of the softer surface and velocity normalised by $a / R_{0}$ where $a$ is the thermal diffusivity and $R_{0}$ the nominal contact radius (see references (1) and (2) for details) All axes are logarithmic, and span a wide range - from very light to very heavy pressure, and from very slow to very fast sliding velocities. Plotted on the map are contours showing the bulk temperatures (full lines) and flash temperatures (dotted lines) that are caused by any given combination of pressure and velocity. The temperature in ${ }^{\circ} \mathrm{C}$, associated with the contours are listed on the lower lef $t$; the lowest contour in each set corresponds to the lowest of these temperatures: the rest follow in sequence. The box in the top left shows the configuration (eq. "Ball on Flat"), the materials of slider and disk, the nominal contact radius $R_{0}$, the asperity size, $R_{a}$, and the ambient temperature. Listed at top right are the hardness, $\mathrm{H}_{0}$, the thermal conductivity, $k$, the thermal diffusivity, a, the coefficient of friction, $\mu_{0}$, and its velocity dependence, two heat diffusion lengths, $L_{1 b}$ and $L_{2 b}$, and the asperity $\|$ ifetime, $n$.

The precedure is fast and flexible: it properly accounts for difference in material properties between the two surfaces, and it includes a realistic treatment of the asperity size and lifetime - a particularly difficult problem $(1,2,3)$.

\section{A SOFT WEAR-TISSTER}

A novel wear pin-on-disk test-rig using low-inertia pneumatic loading has been built. Measurements of dynamic load fluctuations caused by slight misalignment of the disk or by non-planarity of the disk face have been characterized: under pneumatic-control these are greatly reduced. Wide speed range (from 0.001 to $10 \mathrm{~m} / \mathrm{s}$ ) is possible, retaining precision, using contemporary stepper-motor technology. The rig is described in the report (5). All the data presented in references (1), (2) and (3) were obtained using this rig. 


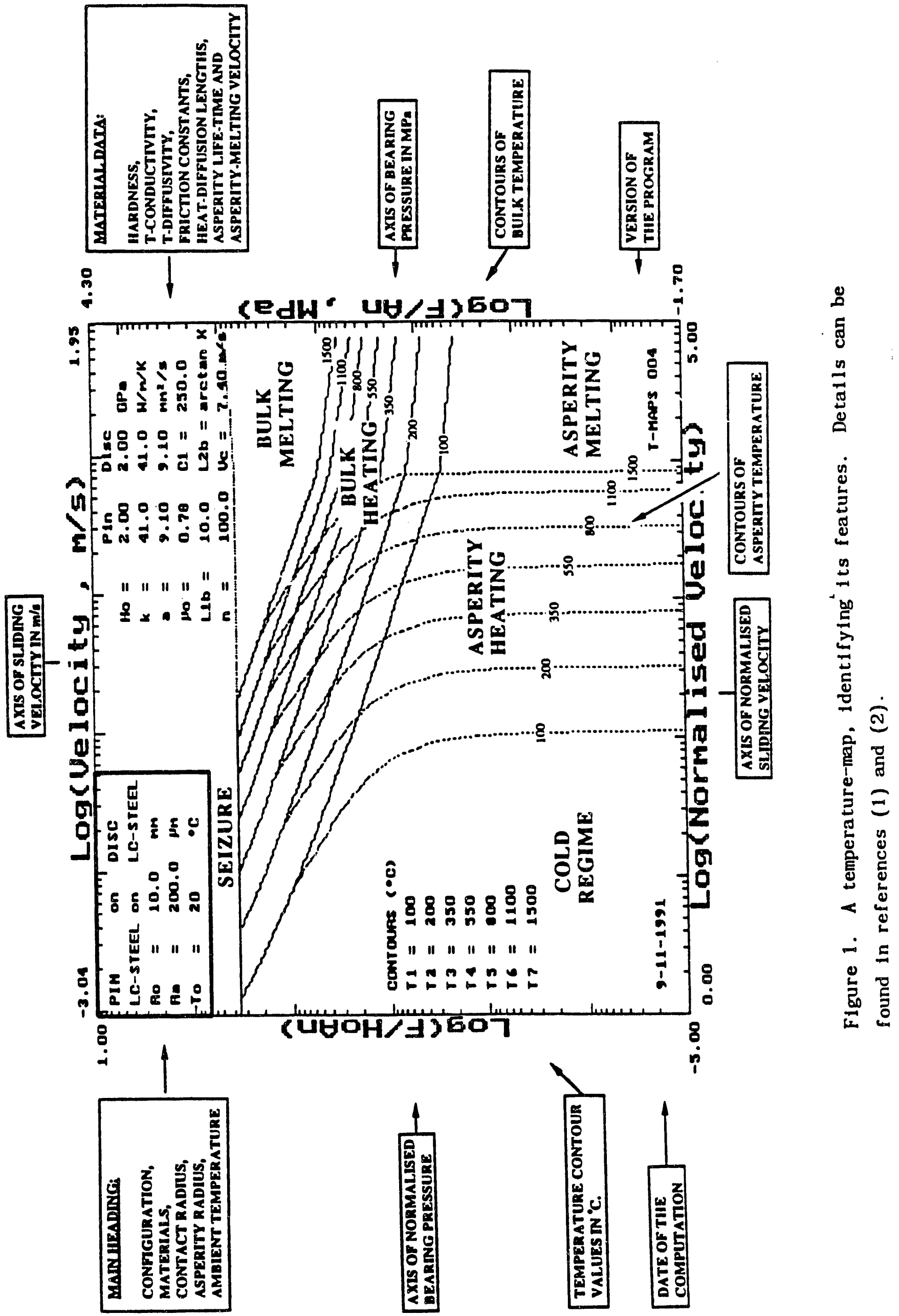




\section{BROAD SURVEY OF WEAR MECHANISTS IN BRITTLE SOLIDS}

Briefly, the observations relating to wear mechanisms in brittle solids are as follows. Sources are listed in reference (3).

At ambient temperature brittle solids, loaded in tension, deform elastically up to fracture. When loaded in compression, many show limited plasticity. But despite this, when contact loads are sufficiently high, surface cracks initiate and propagate to form either a cone (or "Hertzian") crack at a static contact or a chevron-type crack normal to the sliding direction at a sliding contact. The Hertzian crack is caused by the tensile hoop stress around the contact area. When a slider moves, the frictional force reduces the tensile stress in front of the contact, but increases it at the rear, and it is here that cracks nucleate. The friction force thus plays an important role in governing the crack pattern and the consequent wear in ceramics and brittle polymers. For a polycrystalline ceramic (as opposed to glassy solids), grain boundaries are sites for crack propagation because of their lower fracture toughness: and voids, or impurities can also lead to intergranular fracture, giving "grain pull-out". Once cavities have been produced on the surface, "edgespalling" can account for continued formation of wear debris which is a characteristic of ceramic wear in the brittle regime. Elastic or thermal mismatch between grains can increase the possibility of brittle crack propagation at a grain boundary.

Though ceramics normally deform in an almost elastic manner, they can show limited plasticity under a compressive load, or under high-temperature conditions. When a contact causes extensive plastic deformation at the surface, the crack patterns are different from those at an elastic contact. Several models for initiating and propagating these cracks exists. Lateral cracking around the plastic deformation zone caused by residual stress, or by pile-ups at grain boundaries have been suggested as causes for crack initiation.

Though most ceramics remain hard at moderate temperatures, the hardness drops when the temperature exceeds about half of the melting point. At high sliding velocities, surface temperatures due to frictional heating can be so high that large-scale surface deformation by plastic flow can occur even in ceramics. The low thermal diffurivities and the high hardness of many ceramics result in high surface temperatures due to frictional 
heating. The temperature difference between the surface and the subsurface generates a compressive stress in the surface and a compensating tensile field some distance below: these superimpose on the mechanical stresses. The combined effects of mechanical and thermal stress fields may then cause thermal cracking at the trailing sides of the asperity. The compressive stress field in the surface may cause localised buckling, creating a spall as sub-surface cracks propagate to the surface. Thermal stress cycling from repeated heating and cooling gives thermal fatigue and causes a cyclic thermo-elastic mismatch between grains which helps a crack to initiate and propagate. When the surface temperature is high enough, oxidation of non-oxide ceramics, or structural transformations and surface melting can occur, affecting both wear and friction.

Reference (3) assembles and reexpresses, in a single terminology, the models for these mechanisms. All depend on contact pressure and sliding velocity; for many, these variables enter through their effect on surface temperature. This allows the final step: the construction of an approximate wear-mechanism map.

\section{TEAR-HECHANISI MAPS}

Wear mechanisms of brittle solids, as explained above, include wear by brittle fracture, wear by thermo-mechanical fracture, and wear by the local and general melting. Under given sliding conditions, one mechanism dominates, but a change in the load or sliding velocity can cause a transition. Al though the models for these mechanisms cannot describe wear-rates accurately, they provide an overall framework for characterising ceramic wear, identifying the dominant wear mechanisms under given conditions, and the location of the transitions. The second figure shows an example: it is a wear map for alumina, constructed by positioning wear mechanisms on a temperature map, using the arguments outlined above, and detailed in references (1), (2) and (3), and relies also on the experimental results of the rig described in Section 3 and reference (5), which are used to establish the constants in the wear equations.

The figure illustrates the following features. At low loads and sliding speeds, where the surface temperature remains "cold", wear takes place by micro-scale fracture at asperities. In this regime, labelled MICRO-BRITTLE WEAR, damage accumulates slowly, allowing an induction period 


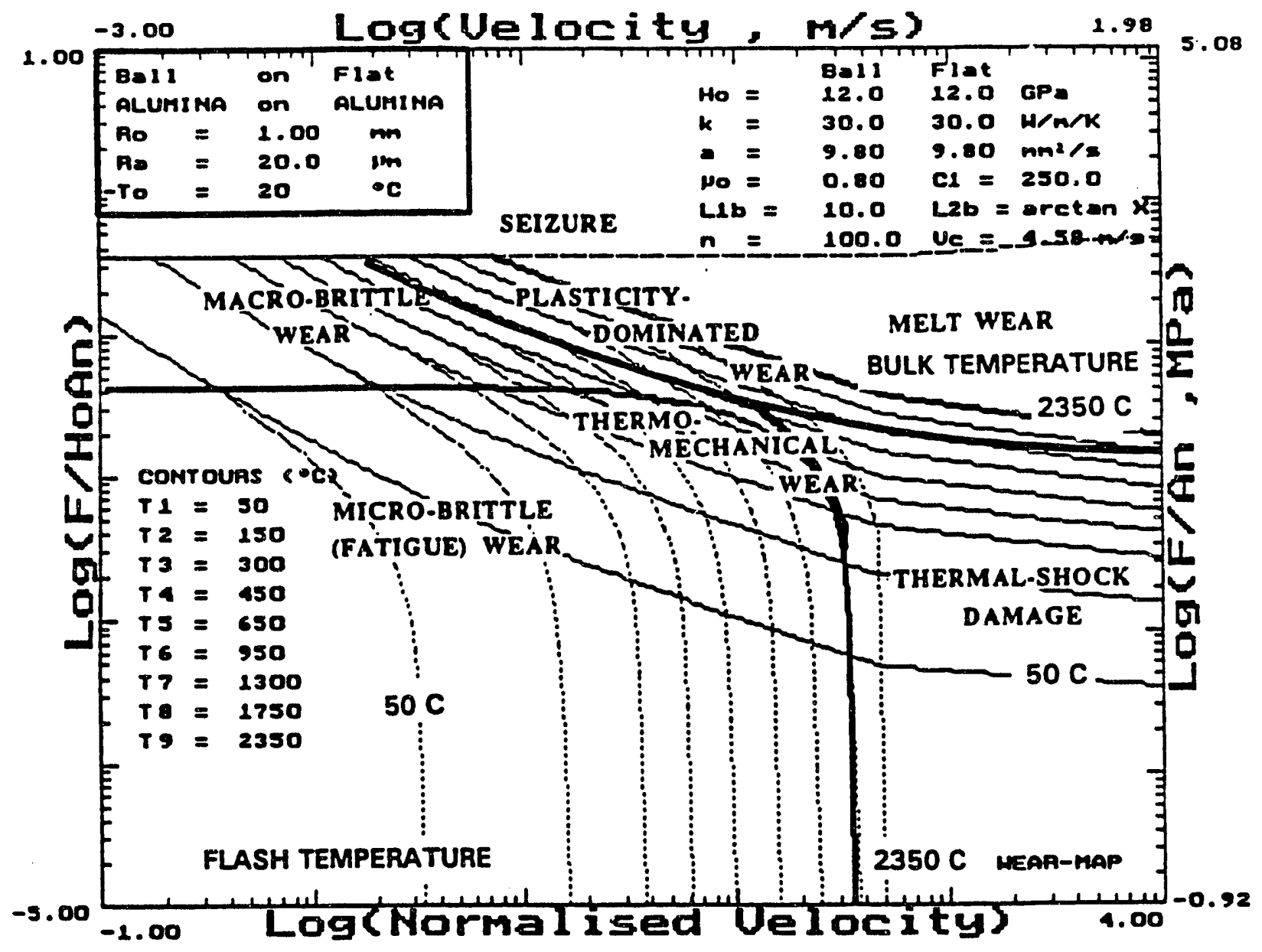

Figure 2. A wear-mechanism map for an alumina ball sliding on an alumina disk, showing the regimes of contact pressure and sliding velocity occupied by each mechanism. The map is based on models (heavy lines) and on experiments (used to set the parameters which enter the models). Details are given in reference (3). 
before measurable wear occurs. As the load increases above a critical level, surface $f$ laws provide nuclei for macro-cracking and wear is dominated by MACRO-BRITTLE FRACTURE. Under smaller loads or in initially macro-defect-free ceramics, micro-scale damage accumulates with the number of sliding passes until it reaches a scale that is picked up by the macro stress field. This incubation period for the appearance of macrocracks resembles a fatigue process, leading ultimately to macro-brittle wear. When the coefficient of friction is low and the contact pressure is high, the contact becomes plastic and SEIZURE may take place. As the sliding speed increases, surface temperatures at both local and bulk contact areas are raised by frictional heating, giving thermal stresses which superimpose on the mechanical ones, causing a thermo-mechanical wear. At the boundary of the asperity-melting regime, the thermal stress dominates over the mechanical stress and causes THERMAL-SHOCK DAMAGE (asperity-scale cracking by the thermal stresses) even at a low contact pressure, suggesting "thermal fatigue". In the bulk-heating regime, material properties are affected by the elevated temperatures, the hardness falls, and wear is PLASTICITY-DOMINATED. When the bulk temperatures reach the melting point of one of the two surfaces, the mechanism of MELT WEAR becomes dominant.

The figure shows characteristic boundaries around which each wear mechanism competes with others. No claim is made that this map is complete or particularly accurate; but it does bring together a great deal of information and gives a good description of experimental observations.

\section{DDLIVERABLES : PUBLICATIONS AND SOFTIARE}

\section{(a) PUBLICATIONS}

(1) "Temperature Maps for Frictional Heating by Dry Sliding", Ashby, M.F., Abulawi, J. and Kong, H.S., STLE Tribology Transactions, 34, 577 (1991).

(2) "Frictional-Heating Maps and their Applications", Kong, H.S. and Ashby, M.F., MRS Bulletin Uctober (1991), p. 43.

(3) "Wear Mechanisms in Brittle solids", Kong, H.S. and Ashby, M.F., Acta Metall. et Mat. 40, (1992).

(4) "Case Studies in the Application of Temperature Maps for Dry Sliding". Kong, H.S. and Ashby, M.F., Cambridge University Engineering Department Report, CUED/C-MATS/TR.186, February $1 \subseteq 91$. 
(5) "Design and Use of an Instrumented Wear Rig with Pneumatic Loading", Kong, H.S. and Ashby, M.F., Cambridge University Engineering Department Report, CUED/C-MATS/TR. 202.

\section{(b) SOFTWARE}

T-MAPS 4.0 Sof tware, wi th Operating Manual and Background Reading booklets, by M.F. Ashby, H.S. Kong and J. Abulawi, Cambridge University Engineering Department, Cambridge, U.K. 1992. 


\section{HIGILIGTS}

1. The calculation of surface-temperatures - both the "bulk" and the "flash" temperatures - has been simplified and refined. Sof tware (T-MATS 4.0) has been developed which allows rapid calcuation of these temperatures for any pair of materials, differing in properties, in a range of geometries.

2. A pneumatically-loaded wear-rig has been developed which reduces problems associated with dynamic loads.

3. Observations of the mechanisms; of wear in brittle solids have been surveyed; tests using the new rig have extended these observations and helped to isolate identifiable mechanisms.

4. The temperature calculations, the observations and the models have been combined to construct a wear-mechanism map for a ceramic. It illustrates the method.

5. A set of case studies illustrate how the research helps understand very diverse aspects of friction and wear. 

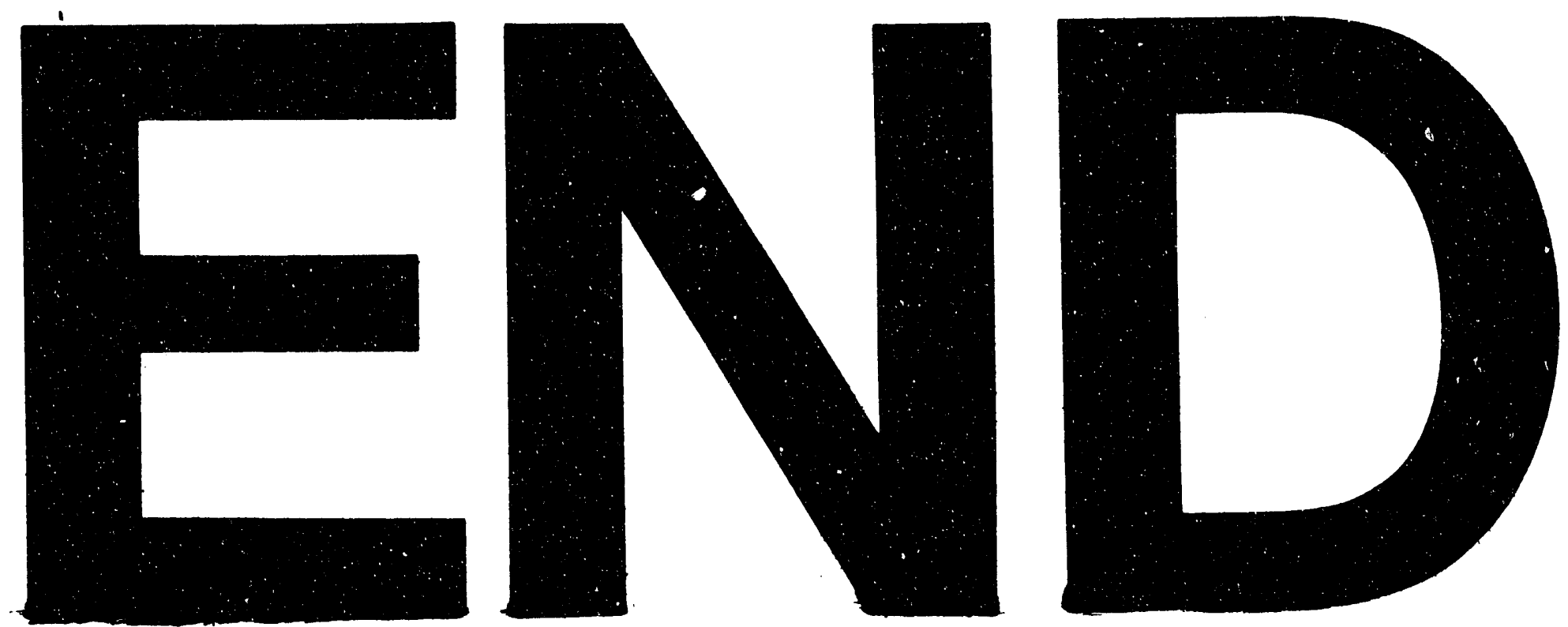

E
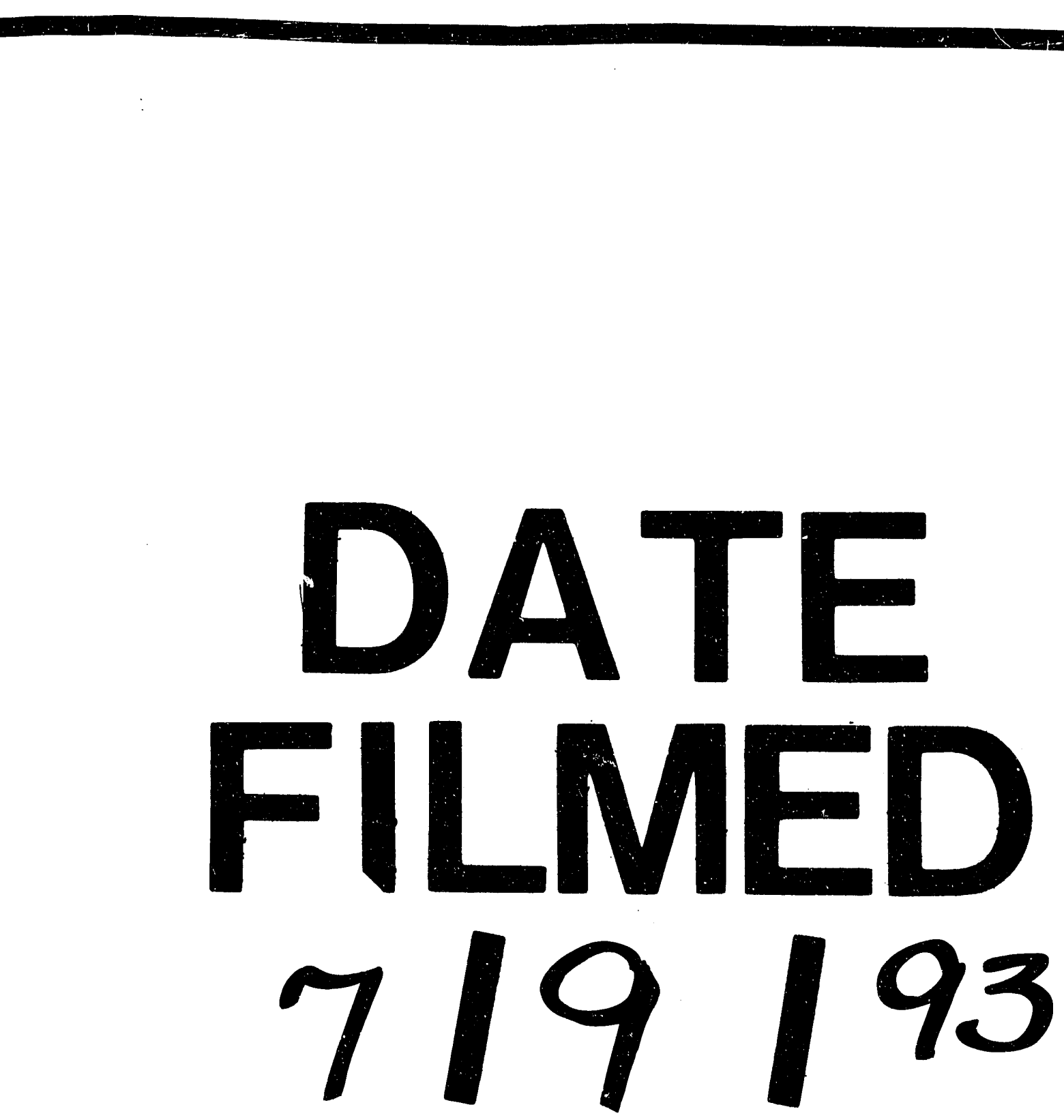